\title{
Assessment of Ileocolonic Inflammation in Crohn's Disease: Which Surrogate Marker Is Better-MaRIA, Clermont, or PET/MR Index? Initial Results of a Feasibility Trial
}

\author{
Yan $\mathrm{Li}^{1}$, Jost Langhorst ${ }^{2}$, Anna Katharina Koch ${ }^{2}$, Aydin Demircioglu ${ }^{1}$, Felix Nensa ${ }^{1}$, Julian Kirchner ${ }^{3}$, \\ Karsten Beiderwellen ${ }^{1,4}$, Onofrio Catalano ${ }^{5}$, Michael Forsting ${ }^{1}$, Ken Herrmann ${ }^{6}$, and Lale Umutlu ${ }^{1}$ \\ ${ }^{1}$ Department of Diagnostic and Interventional Radiology and Neuroradiology, University Hospital Essen, University of Duisburg- \\ Essen, Essen, Germany; ${ }^{2}$ Department of Internal and Integrative Medicine, Kliniken Essen-Mitte, University of Duisburg-Essen, \\ Essen, Germany; ${ }^{3}$ Department of Diagnostic and Interventional Radiology, University Hospital Dusseldorf, Düsseldorf, Germany; \\ ${ }^{4}$ Medizinisches Versorgungszentrum Prof. Dr. Uhlenbrock and Partner, Dortmund, Germany; ${ }^{5}$ Abdominal Imaging and Martinos \\ Center for Biomedical Imaging Research, Department of Radiology, Massachusetts General Hospital, Harvard Medical School, \\ Boston, Massachusetts; and ${ }^{6}$ Department of Nuclear Medicine, University Hospital Essen, University of Duisburg-Essen, Essen, \\ Germany
}

Our objective was to define an ${ }^{18}$ F-FDG PET/MR enterography index as a hybrid surrogate marker for active ileocolonic inflammation in Crohn's disease (CD) and assess its diagnostic performance in comparison to validated MR indices (MR index of activity [MaRIA], Clermont score). Methods: Fifty-two CD patients with recurrent symptoms underwent ileocolonoscopy and ${ }^{18} \mathrm{~F}$-FDG PET/MR enterography. Three hundred three ileocolonic segments were assessed for inflammation using MaRIA and the Clermont score as well as the newly defined PET/MR index. On the basis of tobit regression, the $\mathrm{PET} / \mathrm{MR}$ index was defined as $(0.87 \times$ wall thickness $)+(1.97 \times$ edema $)+(0.83 \times$ ulceration $)+(0.55 \times$ SUVmax ratio $)+1.14$. The endoscopic activity of inflammation was determined by the simplified endoscopic activity score for CD (SES-CD). Receiver-operating-characteristic curves for each surrogate marker were created and tested against each other using the DeLong test, and diagnostic accuracies were compared using the McNemar test. Correlations between surrogate markers and SES-CD were tested with the Spearman rank correlation test. Results: The PET/MR index showed a comparable sensitivity but a significantly higher specificity and accuracy than MaRIA and the Clermont score in predicting both active and severe inflammation (active inflammation: specificities of $0.933,0.711$, and 0.707 and accuracies of $0.921,0.739$, and $0.736, P<0.001$; severe inflammation: specificities of $0.91,0.81$, and 0.785 and accuracies of $0.914,0.818$, and $0.795, P<0.01$, respectively). All surrogate markers correlated moderately with SES-CD on a segmental basis and a global level $(0.5<\rho<0.7$, all $P<0.001$ ). Conclusion: As a hybrid surrogate marker comprising MR parameters and the PET component, the PET/MR index yielded significantly improved specificity and diagnostic accuracy compared with conventional MR indices (MaRIA and the Clermont score), demonstrating its high potential for noninvasive assessment of $C D$.

\footnotetext{
Received Aug. 15, 2018; revision accepted Oct. 15, 2018.

For correspondence or reprints contact: Yan Li, Department of Diagnostic and Interventional Radiology and Neuroradiology, University Hospital Essen, University of Duisburg-Essen, Hufelandstrasse 55, 45147 Essen, Germany. E-mail: yan.li@uk-essen.de

Published online Nov. 2, 2018.

COPYRIGHT (C 2019 by the Society of Nuclear Medicine and Molecular Imaging.
}

Key Words: PET/MR; inflammatory bowel disease; Crohn's disease; MR enterography; hybrid imaging

J Nucl Med 2019; 60:851-857

DOI: 10.2967/jnumed.118.216937

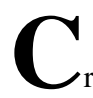
and has been well established as a noninvasive imaging tool in the management of Crohn's disease (CD) from the initial diagnosis to the staging of the disease, as well as throughout its entire course to monitor follow-up (1).

The MR index of activity (MaRIA) and Clermont score are 2 well-known and widely validated multiparametric MR indices that have been proven to provide high diagnostic accuracies in assessing inflammatory activity and the severity of CD (2-7). The Clermont score was derived from MaRIA and replaced the acquisition of contrast-enhanced imaging (as required for MaRIA) with diffusion-weighted imaging (DWI) (7). Thus, given the recent controversial discussions about the repetitive application of MR contrast agents and the potential association with gadolinium deposition in the brain (8), the Clermont score may offer favorable benefits for repetitive MRI in CD patients. Furthermore, neither bowel cleansing nor rectal enema is necessary for the Clermont score (9), significantly increasing patient comfort.

Over the past few years, several studies have investigated and demonstrated the ability of ${ }^{18} \mathrm{~F}$-FDG PET and ${ }^{18} \mathrm{~F}$-FDG PET/CT to reliably detect moderate to severe inflammation in CD (10-12). Thus far, only a limited number of studies have investigated the feasibility and clinical utility of PET/MRI in CD, and most of them compared the diagnostic accuracies and clinical impact of each submodality alone (13-16). Hence, it is of great clinical interest and importance to investigate whether hybrid PET/MR parameters, incorporating both PET and MR, exhibit higher diagnostic value than MR indices alone.

Therefore, the aim of this study was to define a new PET/MR surrogate marker and compare the diagnostic performance of different MR and PET/MR surrogate markers in assessing inflammatory activity 
and severity in patients with $\mathrm{CD}$ in comparison to the endoscopic reference standard.

\section{MATERIALS AND METHODS}

\section{Patients}

The study was approved by the institutional review board (number 11-4824-BO), and written informed consent was obtained from all patients. From October 2014 to September 2017, 53 patients were enrolled in this single-center study. The eligibility criteria included an established diagnosis of $\mathrm{CD}$ (based on clinical, radiologic, and endoscopic criteria), an age of at least $18 \mathrm{y}$, and recurrent clinical symptoms such as diarrhea or abdominal pain. Exclusion criteria were contraindications to MR, severe renal failure (glomerular filtration rate $<30 \mathrm{~mL} / \mathrm{min}$ ), and primary sclerosing cholangitis. Primary sclerosing cholangitis was considered an exclusion criterion because inflammation of the bile ducts might lead to an overall increased hepatic tracer uptake in PET, introducing a bias in the ratio of bowel segment uptake to liver uptake. Ileocolonoscopy was performed before the PET/MR scan in 49 patients at an average interval of $3 \mathrm{~d}$ (maximum, $14 \mathrm{~d}$ ). The remaining 4 patients underwent the PET/MR examination 1-3 d ahead of endoscopy. One patient could not complete the PET/MR scan because of severe claustrophobia and was excluded from the analysis.

\section{Ileocolonoscopy Procedures and Evaluation}

Endoscopic findings with biopsy were considered the reference standard for evaluation of disease activity and severity. Ileocolonoscopy was performed under anesthesia by an endoscopist with more than $10 \mathrm{y}$ of experience using a video colonoscope (CF-Q $165 \mathrm{~L}$; Olympus). For bowel cleansing, an electrolyte solution (Klean-Prep; Helsinn) was ingested on the evening and the morning before the endoscopic examination. Ileocolonoscopy was performed under sedation (100-300 mg of propofol; Fresenius). The ileocolon was divided into 7 segments: terminal ileum, cecum (ileocecal valve to $15 \mathrm{~cm}$ distant from the ileocecal valve), ascending colon, transverse colon, descending colon, sigmoid, and rectum. The location, severity, and extent of inflammation were evaluated on the basis of the simplified endoscopic activity score for CD (SES-CD) (17). Furthermore, the degree of inflammation in each segment was also categorized, as follows: absence of inflammation (category 0 ), active disease (SES-CD $\geq 2$ ) (category 1 ), or severe inflammation with the presence of superficial or deep ulcerations (category 2). A global SES-CD score was determined as the sum of each segmental SES-CD.

\section{PET/MR Enterography and Imaging Protocol}

PET/MR examinations were performed on an integrated 3.0-T PET/ MR scanner (Biograph mMR; Siemens Healthcare). As the luminal distension of colonic segments obtained by MR enterography is inherently sufficient in most cases and rectal enema is commonly not well tolerated by patients, no rectal enema was administered in this study. Patient preparation comprised a fasting period of $6 \mathrm{~h}$ before the $\mathrm{PET} / \mathrm{MR}$ examination as well as the ingestion of $2,000 \mathrm{~mL}$ of an oral contrast agent containing $2.5 \%$ mannitol and $0.2 \%$ locust bean gum (Roeper) $45 \mathrm{~min}$ before the examination. A body-weight-adapted dose $\left(2 \mathrm{MBq} / \mathrm{kg}\right.$ ) of ${ }^{18} \mathrm{~F}-\mathrm{FDG}$ (mean, $150 \pm 22 \mathrm{MBq}$ ) was administered intravenously $1 \mathrm{~h}$ before the scan. Patients were placed prone for reduction of the anterior-posterior scan range as well as motion artifacts. A $20 \mathrm{mg}$ dose of scopolamine (Buscopan; Boehringer Ingelheim) was injected intravenously to further minimize motion artifacts before the acquisition of dynamic contrast-enhanced T1-weighted sequences. For PET datasets, 3 bed positions, each of which took approximately $8 \mathrm{~min}$, were obtained for coverage of the entire abdomen and pelvis. PET images were reconstructed using 3-dimensional ordinary Poisson ordered-subset expectation maximation ( 3 iterations, 21 subsets, $2.1 \times$ $2.1 \times 2.0 \mathrm{~mm}$ voxels, 3-dimensional gaussian filter of $4.0 \mathrm{~mm}$ ). The obtained MR sequences, with technical details, are listed in Supplemental Table 1 (supplemental materials are available at http:// jnm.snmjournals.org).

\section{Imaging Analysis}

MRI analysis was performed in consensus by 2 physicians with 4 and $7 \mathrm{y}$ of MR experience in abdominal and hybrid imaging, as well as by a nuclear medicine physician, using a dedicated postprocessing workstation (Universal Viewer 6.0; GE Healthcare). All 3 physicians were masked to the endoscopy results. Dedicated postprocessing software for hybrid imaging (Syngo.via, VB10B; Siemens Healthcare) was used to measure the SUVs of each segment and liver in order to calculate the $S U V_{\text {max }}$ ratio $\left(\mathrm{SUV}_{\max }\right.$ of segment relative to $S U V_{\text {max }}$ of liver). The $\mathrm{SUV}_{\max }$ of the bowel segments was measured by placing a spheric volume of interest (mean size, $8.09 \pm 3.4 \mathrm{~cm}^{3}$ ) in the most thickened part of each segment. The $\mathrm{SUV}_{\max }$ of the liver was calculated using a larger spheric volume in the right lobe of the liver (mean size, $48.4 \pm 21.37 \mathrm{~cm}^{3}$ ).

In accordance with the endoscopic analysis, 7 ileocolonic segments were subdivided for the PET/MRI and MRI analysis.

The MaRIA score was defined as $(1.5 \times$ wall thickness $)+(0.02 \times$ relative contrast enhancement $)+(5 \times$ edema $)+(10 \times$ ulceration $)(3)$. Wall thickness was measured in millimeters, and the signal intensity of the bowel wall in the fat-suppressed T1-weighted 3-dimensional volumetric interpolated breath hold examination was measured by placing a region of interest in the most thickened part. As described in the original article on MaRIA (4), the signal intensity of the wall was determined as the average of 3 measurements. Relative contrast enhancement was calculated according to the publication by Semelka et al. (18).

As published by Hordonneau et al., the Clermont score was calculated as $(1.646 \times$ bowel thickness $)-(1.321 \times$ apparent diffusion coefficient $)+(5.613 \times$ edema $)+(8.306 \times$ ulceration $)+$ $5.039(5,7)$. The mean apparent diffusion coefficient of each segment was calculated as an average of 3 measurements with a region of interest (mean size, $0.45 \pm 0.25 \mathrm{~cm}^{2}$ ) in the apparent diffusion coefficient map.

A global MaRIA index was defined as the sum of each MaRIA score from 7 ileocolonic segments. A total Clermont score was defined in the same way.

To investigate the diagnostic potential of hybrid surrogate markers, a new and simplified PET/MR index was defined. A tobit regression was calculated to predict the SES-CD score based on the 3 wellknown independent variables shared in common by both MR indices, namely wall thickness, edema, and ulceration, as well as $\mathrm{SUV}_{\text {max }}$ ratio as the PET component, since the variable $\mathrm{SUV}_{\max }$ ratio is known to facilitate a better diagnostic performance than $\mathrm{SUV}_{\text {max }}$ measurements of the bowel segments and correlates strongly with SES-CD (15). The analysis of our study showed that $\mathrm{SUV}_{\max }$ ratio was highly significant in predicting SES-CD score $(P=0.009)$. Bootstrapping with 1,000 repeats was used to prevent the model from overfitting. The final PET/MR index was derived from this regression and had the following form: PET/MR index $=(0.87 \times$ wall thickness $)+(1.97 \times$ edema $)+(0.83 \times$ ulceration $)+(0.55 \times$ SUVmax ratio) +1.14 .

\section{Statistical Analysis}

The diagnostic performance of MaRIA, Clermont score, and PET/ MR index in detecting active inflammation was tested by creating receiver-operating-characteristic curves with a calculated area under the curve (AUC). An optimal cutoff for the PET/MR index was determined by the maximum value of the Youden index. Second, for 
detecting segments with severe inflammation with endoscopic ulceration, receiver-operating-characteristic curves and optimal cutoffs were calculated in a similar fashion. The differences in the receiveroperating-characteristic curves of all the surrogate markers were tested against each other by the DeLong test. According to predefined cutoffs for both MR indices and an optimal cutoff for the PET/MR index, the sensitivities, specificities, and accuracies for each index in detecting active disease and severe inflammation were compared with each other using the McNemar test. The Mann-Whitney $U$ test was performed to compare the median values of all 3 indices between segments with mild to moderate nonulcerative inflammation and segments with severe ulcerative inflammation.

Furthermore, correlations between segmental or global SES-CD scores and all the surrogate markers were tested by the Spearman rank correlation test.

All statistical tests were performed with SPSS (version 23; IBM) and the R-Software environment for statistical computing (version 4.3; R Foundation for Statistical Computing). $P$ values were adjusted for multiple testing using the Holm-Bonferroni method. A $P$ value of less than 0.05 was considered significant.

\section{RESULTS}

\section{Patient Characteristics}

Because of previous surgical resections in 16 patients, 303 bowel segments (46 ileum and 257 colon) were analyzed based on endoscopy and cross-sectional PET/MRI. Active inflammation (defined as SES-CD $\geq 2$ ) was found in 47 segments (19 ileum and 28 colon), of which 14 segments (6 ileum and 8 colon) showed severe inflammation with ulcers. Active inflammation was endoscopically confirmed in 27 of 52 patients. Demographic and clinical data are summarized in Table 1.

\section{Diagnostic Performance of MR Indices and PET/MR Index in Detecting Active Inflammation}

A segmental SES-CD score of at least 2 was considered active inflammation in our study. Using the predefined cutoff of 7 for predicting active inflammation (3), MaRIA yielded a sensitivity of 0.894 and specificity of 0.711 . Using the predefined cutoff of 8.4 (9), the Clermont score performed comparably well, with a sensitivity of 0.894 and a specificity of 0.707 . Confirmed by the DeLong test, both MR indices were not significantly different in their receiver-operating-characteristic curves (AUC, 0.916 for MaRIA and 0.914 for Clermont score, $P>0.05$, respectively). Using an optimal cutoff of 6.85 , the sensitivity of the PET/MR index was comparable to MaRIA and Clermont score (0.851 vs. both 0.894 , $P>0.05$, respectively), whereas specificity was significantly higher than both MR surrogate markers (0.933 vs. 0.711 and 0.707, $P<0.001$, respectively). No significant differences in AUC were found among PET/MR index, MaRIA, and Clermont score $(0.924,0.916$, and 0.914 , all $P>0.05$, respectively). The AUC of $\mathrm{SUV}_{\text {max }}$ ratio alone (0.857) was significantly lower than that of all 3 multiparametric indices (all $P<0.05$ ), and an optimal cutoff of 1.32 was chosen. The diagnostic performance of PET/ MR and MR indices for predicting active inflammation is summarized in Table 2 and Figure 1.

\section{Diagnostic Performance of MR Indices and PET/MR Index in Detecting Severe Inflammation with Ulcers}

Fourteen inflammatory segments with superficial or deep ulcers were endoscopically diagnosed in our study (Fig. 2). MaRIA yielded a sensitivity of 1.00 and a specificity of 0.810 using the predefined cutoff of 11 (3). Again, Clermont score showed similar characteristics in predicting severe inflammation, with a sensitivity of 1.00 and a specificity of 0.785 by a predefined cutoff of 12.5 (9).

Both MR indices performed very similarly, and the DeLong test revealed no significant difference in their AUC (0.962 for MaRIA, 0.970 for Clermont score, $P>0.05$, respectively). Using the PET/ MR index, an AUC of 0.971 with a sensitivity of 1.00 and a specificity of 0.910 (cutoff of 8.95 ) could be obtained. Comparable to the detection of active disease, the PET/MR index yielded the same sensitivity yet significantly higher specificity over both MR indices $\left(P<0.05\right.$, respectively). The AUC of $\mathrm{SUV}_{\max }$ ratio alone (0.935) was again lower than that of all 3 multiparametric indices but without a significant difference (all $P>0.05$ ), and the optimal cutoff was defined as 2.16. The diagnostic performance for predicting severe inflammation is summarized in Table 3 and Figure 3.

\section{Comparison of 3 Indices Between Segments with Mild to Moderate Inflammation Without Ulceration and Segments with Severe Ulcerative Inflammation}

According to the Mann-Whitney $U$ test, the median values of MaRIA, Clermont score, and PET/MR index were significantly

TABLE 1

Patients' Sociodemographic and Clinical Characteristics

\begin{tabular}{|c|c|}
\hline Characteristic & Data \\
\hline Age $(y)$ & $43.5 \pm 13.11(20-67)$ \\
\hline \multicolumn{2}{|l|}{ Sex } \\
\hline Female & $34(64.2)$ \\
\hline Male & $19(35.8)$ \\
\hline \multicolumn{2}{|l|}{ Location (Montreal) } \\
\hline L1 (ileal) & $32(60.4)$ \\
\hline L2 (colonic) & $6(11.3)$ \\
\hline L3 (ileocolonic) & $14(26.4)$ \\
\hline Missing & $1(1.9)$ \\
\hline Time since diagnosis $(\mathrm{y})$ & $13.94 \pm 11.22(2-46)$ \\
\hline Smokers & $6(11.3)$ \\
\hline Surgery history & $16(30.8)$ \\
\hline \multicolumn{2}{|l|}{ Blood values } \\
\hline C-reactive protein & $1.09 \pm 1.79(0.1-10)$ \\
\hline $\begin{array}{l}\text { Blood } \\
\quad \text { sedimentation rate }\end{array}$ & $9.83 \pm 14.19(2-74)$ \\
\hline Leukocytes & $9,257.26 \pm 15,327.05(1,095-116,900)$ \\
\hline Thrombocytes & $284.62 \pm 93.74(154-679)$ \\
\hline $\begin{array}{l}\text { Calprotectin levels } \\
\qquad(\mu \mathrm{g} / \mathrm{g})\end{array}$ & $217.00 \pm 82.34(31.67-372.47)$ \\
\hline \multicolumn{2}{|l|}{ Medication } \\
\hline Steroids & $20(37.7)$ \\
\hline $\begin{array}{l}\text { Thiopurine- } \\
\text { methotrexate }\end{array}$ & $9(17)$ \\
\hline Biologics & 7 (13.2) \\
\hline Mesalamine & $9(17)$ \\
\hline Other & $15(28.3)$ \\
\hline
\end{tabular}

Qualitative data are expressed as number, followed by percentage in parentheses; continuous data are expressed as mean \pm SD, followed by range in parentheses. 
TABLE 2

Diagnostic Performance of MR and PET/MR Indices in Predicting Active Inflammation

\begin{tabular}{|c|c|c|c|c|c|}
\hline Index & AUC & Sensitivity & Specificity & Accuracy & Cutoff \\
\hline MaRIA & 0.916 & 0.894 & 0.711 & 0.739 & 7 \\
\hline Clermont score & 0.914 & 0.894 & 0.707 & 0.736 & 8.4 \\
\hline PET/MR index & 0.924 & 0.851 & 0.933 & 0.921 & 6.85 \\
\hline \multicolumn{6}{|l|}{$P$} \\
\hline MaRIA vs. Clermont & n.s. & n.s. & n.s. & n.s. & \\
\hline MaRIA vs. PET/MR & n.s. & n.s. & $<0.001$ & $<0.001$ & \\
\hline Clermont vs. PET/MR & n.s. & n.s. & $<0.001$ & $<0.001$ & \\
\hline
\end{tabular}

different between segments with different inflammatory severity (all $P<0.05$, Table 4).

\section{Correlations Between Imaging Surrogate Markers and SES-CD}

All surrogate markers correlated moderately with SES-CD both on a segmental basis and on a global level $(0.5<\rho<0.6$, all $P<$ $0.001)$, whereas the correlation coefficients were generally higher on a global level $(0.6<\rho<0.7$, all $P<0.001)$ (Supplemental Tables 2 and 3).

In the 46 ileal segments, Clermont score correlated significantly with MaRIA $(\rho=0.992, P<0.001)$, whereas for the 257 colonic segments the correlation was slightly reduced ( $\rho=0.860, P<0.001$ ). As shown in Figure 4 , the PET/MR index showed a very high correlation with MaRIA $(\rho=0.902$, $P<0.001)$ and Clermont score $(\rho=0.891, P<0.001)$ on a segmental basis.

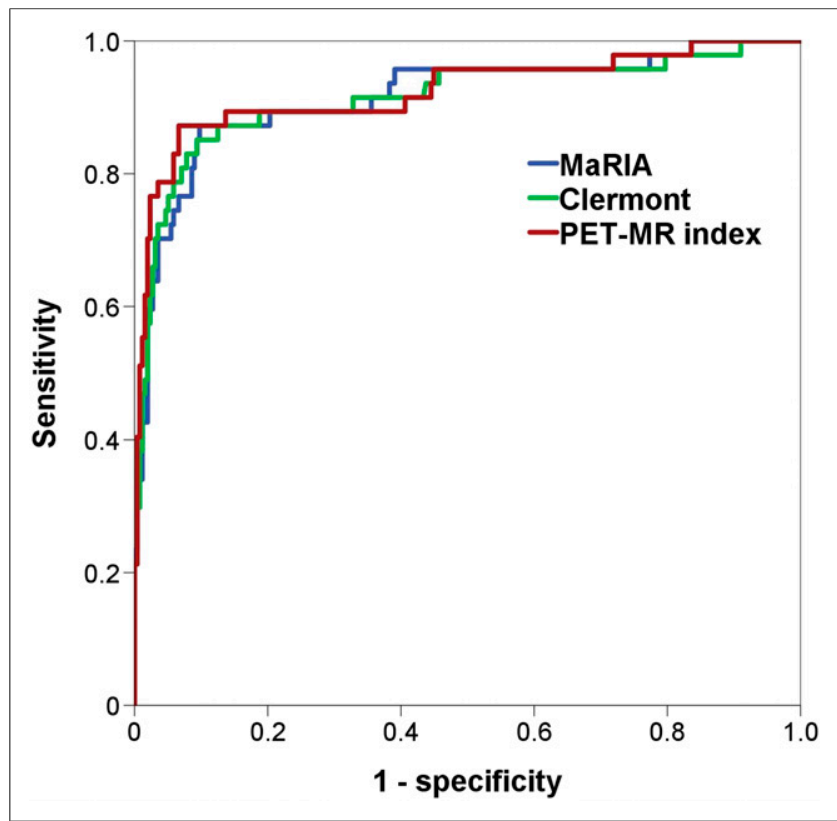

FIGURE 1. Receiver-operating-characteristic curves of PET/MR and MR indices in predicting endoscopically active inflammation.

\section{DISCUSSION}

In this prospective study, the diagnostic accuracy of hybrid and conventional MRI surrogate markers in assessing ileocolonic inflammation was compared against endoscopy in patients with CD. Our results confirm previous retrospective studies in demonstrating the high correlation and comparable diagnostic accuracy of MaRIA and the Clermont score. Furthermore, our study underlines the high diagnostic potential of the hybrid PET/MR index in yielding a significantly increased specificity for detection of active inflammation and severe ulcerative inflammation when compared with both conventional surrogate markers (MaRIA and the Clermont score).

The MaRIA and Clermont score systems have been proposed and demonstrated as highly valid MR surrogate markers in several initial derivation and validation studies $(3-5,7)$. In a recently

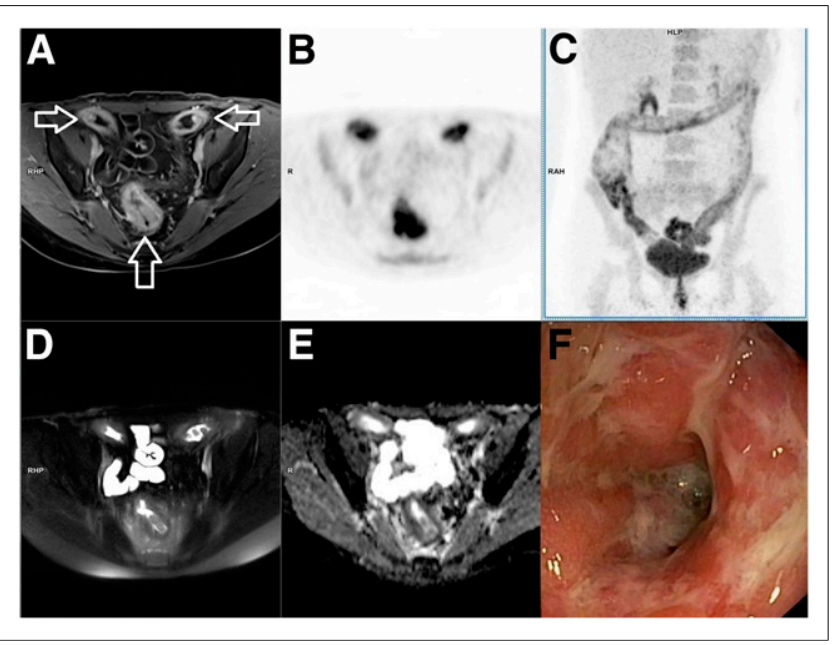

FIGURE 2. Images of 39 -y-old CD patient with severe ileocolonic inflammation confirmed by endoscopy. (A) Axial fat-saturated T1-weighted 3-dimensional volumetric interpolated breath-hold examination in portal venous phase showing increased contrast enhancement in thickened wall and irregular mucosal surface indicating ulcerations (arrows). (B-E) Confirmation of these findings in PET imaging (B), maximum-intensity projection (C), and fat-saturated T2-weighted HASTE imaging (D), as well as in DWI (corresponding apparent diffusion coefficient map (E)). (F) Endoscopic findings of deep ulceration, marked erythema, granularity, and absence of vascular pattern. 
TABLE 3

Diagnostic Performance of PET/MR and MR Indices in Predicting Severe Ulcerative Inflammation

\begin{tabular}{llcccc}
\hline \multicolumn{1}{c}{ Index } & AUC & Sensitivity & Specificity & Accuracy & Cutoff \\
\hline MaRIA & 0.962 & 1.00 & 0.81 & 0.818 \\
Clermont score & 0.970 & 1.00 & 0.785 & 0.795 & 0.914 \\
PET/MR index & 0.971 & 1.00 & 0.910 & n.s. \\
$P$ & & & n.s. & 0.007 \\
MaRIA vs. Clermont & n.s. & n.s. & $<0.001$ & $<0.001$ \\
MaRIA vs. PET/MR & n.s. & n.s. & $<0.001$ & \\
Clermont vs. PET/MR & n.s. & n.s. & & \\
\hline n.s. $=$ not significant. & & & & \\
\hline
\end{tabular}

published retrospective study with 224 ileocolonic segments, MaRIA was shown to offer better operating characteristics than the Clermont score regarding its overall accuracy (2). On the basis of a comparable study setup regarding the definition of disease severity using endoscopy and SES-CD, very similar results could be reproduced in our study in predicting active inflammation for the Clermont score in sensitivity ( 0.89 in our study vs. 0.90 in their study) and specificity ( 0.71 vs. 0.78$)$, as well as in sensitivity (0.89 vs. 0.88 ) for MaRIA. Yet, no significant difference in their diagnostic performance could be found in our study. In detecting severe ulcerative inflammation, again both MR indices performed comparably well in our study, with a sensitivity of 1.0 but slightly reduced specificity ( 0.81 vs. 0.91 in their study for MaRIA and 0.79 vs. 0.89 for the Clermont score). In a prospective study with 207 ileocolonic segments, in which diagnostic accuracies of both MR indices were tested against the presence of superficial or deep ulcerations in endoscopy, MaRIA and the Clermont score were proven to be equally effective (6). The major distinction between MaRIA and the Clermont score is based on the omission of contrast-enhanced imaging and the addition of DWI in the Clermont score. Considering the recent awareness of gadolinium deposition in the brain after repeated administrations, the omission of a contrast agent may be regarded as beneficial, considering the need for repeated applications for MRI-based therapy monitoring in a nononcologic patient cohort. Hence, whereas application of the Clermont score may be favorable in this regard, the use of DWI as a fundamental part of this surrogate marker bears the potential for disadvantages. DWI has been introduced and well established for abdominal imaging over the past decade (19). Nevertheless, it is also well known that DWI is highly susceptible to artifacts and discrepancies related to $\mathrm{B}_{1}$ field inhomogeneities, different field strengths, or different scanner vendors $(20,21)$, potentially impairing the standardized use of surrogate markers such as the Clermont score, which are strictly dependent on predefined cutoffs. All these impediments may be addressed by the application of the newly introduced PET/MR index.

${ }^{18}$ F-FDG PET imaging has been well established for the assessment of ileocolonic CD (10-12), and the reported sensitivities in detecting inflamed segments ranged from $82 \%$ to $90 \%$ and specificities from $89 \%$ to $97 \%$. In a previous study with PET/MR in $\mathrm{CD}$ (15), the $\mathrm{SUV}_{\max }$ ratio was proven to be the most important parameter for predicting endoscopic severe inflammation. However, for detecting active inflammation, the $\mathrm{SUV}_{\max }$ ratio was inferior to MR parameters. With an optimal cutoff of 1.32 , $\mathrm{SUV}_{\max }$ ratio in our study could achieve a sensitivity of 0.766 , specificity of 0.906 , and accuracy of 0.884 . With the added values of MR parameters, PET/MR index performed significantly better than monoparametric $\mathrm{SUV}_{\max }$ ratio in detecting active inflammation (cutoff, 6.85; sensitivity, 0.851; specificity, 0.933; accuracy, 0.921).

Given the well-validated nature of both MR indices, the aim of our study was to investigate the potential added value of a hybrid surrogate marker, defined as the PET/MR index, and to compare its diagnostic value with that of conventional MR surrogate markers, by means of MaRIA and the Clermont score. The PET/MR index combines the diagnostic strength of PET imaging and MRI in integrating the assessment of metabolism by means of the $\mathrm{SUV}_{\max }$ ratio with 3 conventional MR parameters: wall thickness, edema, and ulceration (all 3 shared by MaRIA and the Clermont score). Our results demonstrate that the PET/MR index facilitates correlation equal to SES-CD and sensitivity equal to MaRIA and the

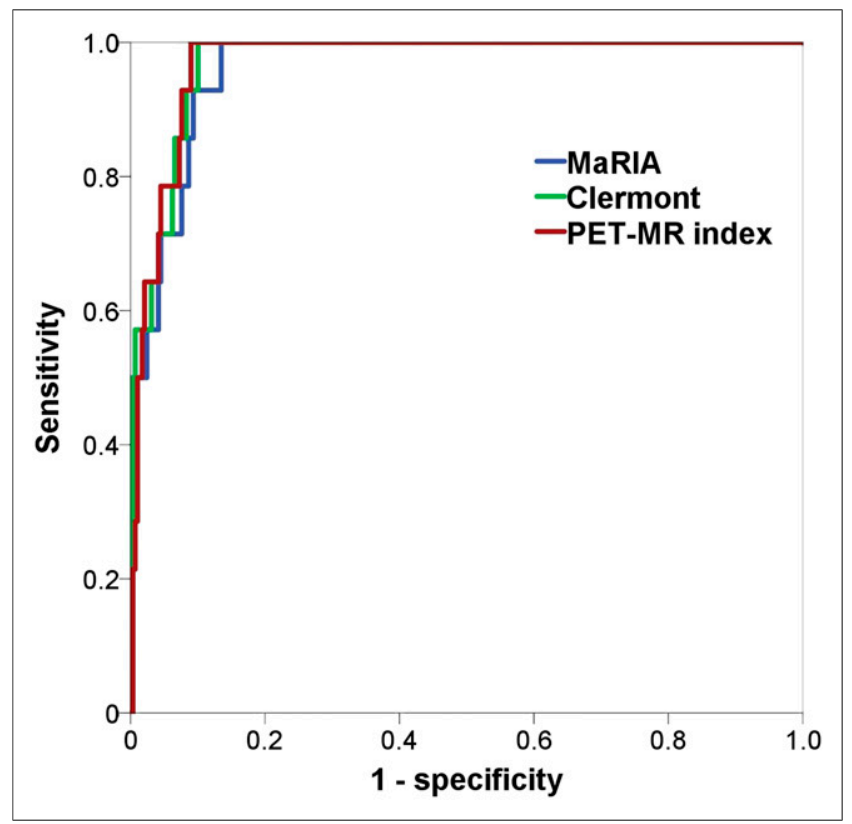

FIGURE 3. Receiver-operating-characteristic curves of PET/MR und $\mathrm{MR}$ indices in predicting severe ulcerative inflammation. 
TABLE 4

Comparison of Median Values of PET/MR and MR Indices Between Segments with Different Degrees of Inflammation

\begin{tabular}{cccc}
\hline Index & $\begin{array}{c}\text { Mild to moderate } \\
\text { nonulcerative } \\
\text { inflammation }\end{array}$ & $\begin{array}{c}\text { Severe } \\
\text { ulcerative } \\
\text { inflammation }\end{array}$ & $P^{*}$ \\
\hline MaRIA & 17.72 & 28.21 & 0.01 \\
\hline $\begin{array}{c}\text { Clermont } \\
\text { score }\end{array}$ & 18.72 & 29.63 & 0.001 \\
\hline $\begin{array}{c}\text { PET/MR } \\
\text { index }\end{array}$ & 9.54 & 12.55 & 0.005 \\
\hline
\end{tabular}

*Mann-Whitney $U$ test.

Clermont score for detection of active and severe inflammation and outperforms both MR indices regarding specificity and diagnostic accuracy, though neither of the indices significantly differs in AUCs.

Compared with ileocolonoscopy, one strength of cross-sectional imaging modalities such as PET/MR enterography is the possibility of assessing the entire gastrointestinal tract. In our study, additional findings that were beyond the range of ileocolonoscopy but had significant clinical implications, such as ileal mesenteric abscesses, were detected in 2 patients, and stenotic lesions in the jejunum or proximal ileum were found in 5 patients.

The main limitation of our study was the relatively low number of inflamed bowel segments. Despite enrolling a reasonably vast patient cohort and analyzing more than 300 ileocolonic segments, only a minority of segments was actively inflamed. This limitation

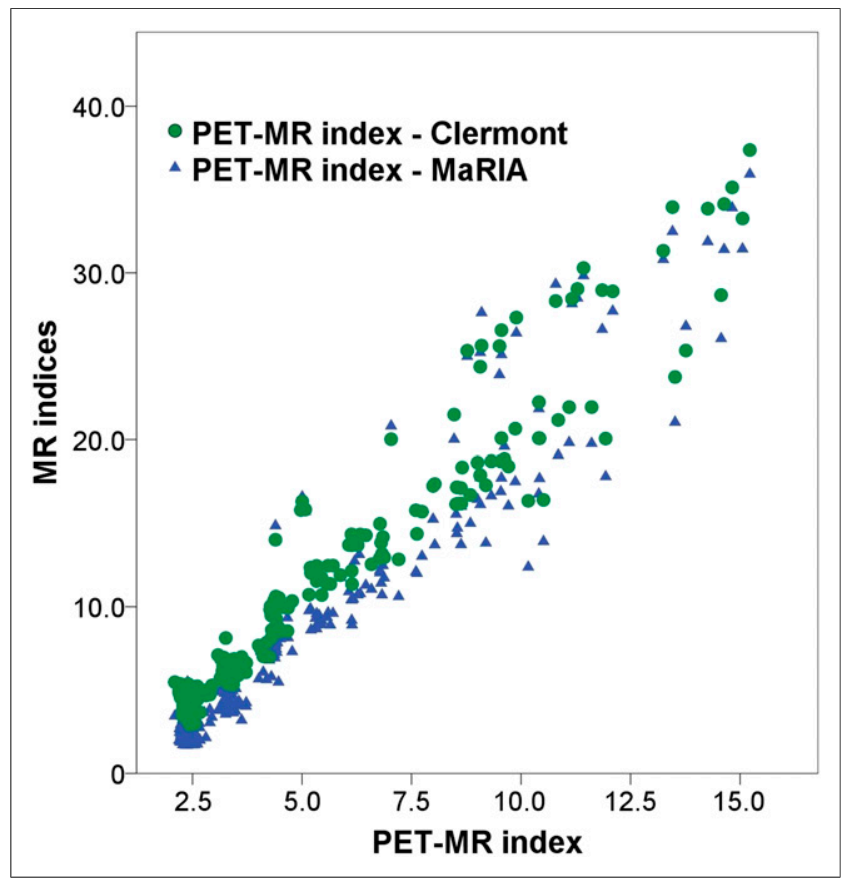

FIGURE 4. Scatterplot indicating high correlation between PET/MR index and MR indices on segmental basis. should be addressed in future multicentric trials to further test the reproducibility and validity of the proposed PET/MR index. Furthermore, in view of the fact that over $90 \%$ of our patients underwent ileocolonoscopy ahead of PET/MR enterography, at an average interval of $3 \mathrm{~d}$, and that antiinflammatory medication is a common treatment in $\mathrm{CD}$, the inflammatory status might have changed during this interval, which might negatively influence the diagnostic accuracies of all 3 surrogate makers. Moreover, because the endoscopic biopsies might also affect the inflammatory status of the bowel wall, for future prospective studies PET/ MR should be conducted before endoscopy. Last, even though PET/MR enterography is associated with only a very low amount of ionizing radiation when compared with PET/CT, the application of radioactively labeled ${ }^{18} \mathrm{~F}-\mathrm{FDG}$ requires consideration and should be well thought out, in particular taking into account the high diagnostic performance of MRI alone. Furthermore, the limited availability and relatively high costs of PET/MR are concerns that need to be considered and evaluated in future trials to fully comprehend its diagnostic value for patient care.

\section{CONCLUSION}

The initial results of our feasibility trial are in line with previous publications in confirming the comparability of the MaRIA and Clermont score and demonstrate the high diagnostic potential of the hybrid PET/MR index for detecting ileocolonic inflammation in patients with known CD and suspected recurrence of disease.

\section{DISCLOSURE}

This study was supported by the German Research Foundation (DFG, Deutsche Forschungsgesellschaft, grant LA 1439/1-1). No other potential conflict of interest relevant to this article was reported.

\section{REFERENCES}

1. Panes J, Bouhnik Y, Reinisch W, et al. Imaging techniques for assessment of inflammatory bowel disease: joint ECCO and ESGAR evidence-based consensus guidelines. J Crohns Colitis. 2013;7:556-585.

2. Rimola J, Alvarez-Cofino A, Perez-Jeldres T, et al. Comparison of three magnetic resonance enterography indices for grading activity in Crohn's disease. J Gastroenterol. 2017;52:585-593.

3. Rimola J, Ordas I, Rodriguez S, et al. Magnetic resonance imaging for evaluation of Crohn's disease: validation of parameters of severity and quantitative index of activity. Inflamm Bowel Dis. 2011;17:1759-1768.

4. Rimola J, Rodriguez S, Garcia-Bosch O, et al. Magnetic resonance for assessment of disease activity and severity in ileocolonic Crohn's disease. Gut. 2009;58: 1113-1120.

5. Buisson A, Joubert A, Montoriol PF, et al. Diffusion-weighted magnetic resonance imaging for detecting and assessing ileal inflammation in Crohn's disease. Aliment Pharmacol Ther. 2013;37:537-545.

6. Buisson A, Pereira B, Goutte M, et al. Magnetic resonance index of activity (MaRIA) and Clermont score are highly and equally effective MRI indices in detecting mucosal healing in Crohn's disease. Dig Liver Dis. 2017;49:1211-1217.

7. Hordonneau C, Buisson A, Scanzi J, et al. Diffusion-weighted magnetic resonance imaging in ileocolonic Crohn's disease: validation of quantitative index of activity. Am J Gastroenterol. 2014;109:89-98.

8. Radbruch A, Weberling LD, Kieslich PJ, et al. Gadolinium retention in the dentate nucleus and globus pallidus is dependent on the class of contrast agent. Radiology. 2015;275:783-791.

9. Hordonneau C, Buisson A, Scanzi J, et al. Diffusion-weighted magnetic resonance imaging in ileocolonic Crohn's disease: validation of quantitative index of activity. Am J Gastroenterol. 2014;109:89-98. 
10. Louis E, Ancion G, Colard A, Spote V, Belaiche J, Hustinx R. Noninvasive assessment of Crohn's disease intestinal lesions with ${ }^{18} \mathrm{~F}-\mathrm{FDG}$ PET/CT. $\mathrm{J}$ Nucl Med. 2007;48:1053-1059.

11. Saboury B, Salavati A, Brothers A, et al. FDG PET/CT in Crohn's disease: correlation of quantitative FDG PET/CT parameters with clinical and endoscopic surrogate markers of disease activity. Eur J Nucl Med Mol Imaging. 2014;41: 605-614.

12. Shyn PB, Mortele KJ, Britz-Cunningham SH, et al. Low-dose ${ }^{18}$ F-FDG PET/CT enterography: improving on CT enterography assessment of patients with Crohn disease. J Nucl Med. 2010;51:1841-1848.

13. Catalano OA, Gee MS, Nicolai E, et al. Evaluation of quantitative PET/MR enterography biomarkers for discrimination of inflammatory strictures from fibrotic strictures in Crohn disease. Radiology. 2016;278:792-800.

14. Catalano OA, Wu V, Mahmood U, et al. Diagnostic performance of PET/MR in the evaluation of active inflammation in Crohn disease. Am J Nucl Med Mol Imaging. 2018;8:62-69.

15. Li Y, Beiderwellen K, Nensa F, et al. $\left[{ }^{18} \mathrm{~F}\right] \mathrm{FDG}$ PET/MR enterography for the assessment of inflammatory activity in Crohn's disease: comparison of different MRI and PET parameters. Eur J Nucl Med Mol Imaging. 2018;45:13821393.
16. Domachevsky L, Leibovitzh H, Avni-Biron I, et al. Correlation of ${ }^{18}$ F-FDG PET/ MRE metrics with inflammatory biomarkers in patients with Crohn's disease: a pilot study. Contrast Media Mol Imaging. 2017;2017:7167292.

17. Daperno M, D'Haens G, Van Assche G, et al. Development and validation of a new, simplified endoscopic activity score for Crohn's disease: the SES-CD. Gastrointest Endosc. 2004;60:505-512.

18. Semelka RC, Shoenut JP, Silverman R, Kroeker MA, Yaffe CS, Micflikier AB. Bowel disease: prospective comparison of $\mathrm{CT}$ and 1.5-T pre- and postcontrast MR imaging with T1-weighted fat-suppressed and breath-hold FLASH sequences. J Magn Reson Imaging. 1991;1:625-632.

19. Kiryu S, Dodanuki K, Takao H, et al. Free-breathing diffusion-weighted imaging for the assessment of inflammatory activity in Crohn's disease. J Magn Reson Imaging. 2009;29:880-886.

20. Sasaki M, Yamada K, Watanabe Y, et al. Variability in absolute apparent diffusion coefficient values across different platforms may be substantial: a multivendor, multi-institutional comparison study. Radiology. 2008;249:624-630.

21. Chen X, Qin L, Pan D, et al. Liver diffusion-weighted MR imaging: reproducibility comparison of ADC measurements obtained with multiple breath-hold, free-breathing, respiratory-triggered, and navigator-triggered techniques. Radiology. 2014;271:113-125. 